\title{
Peroneal tendoscopy
}

\author{
Antonio Marmotti • Mattia Cravino • \\ Margherita Germano • Rainero Del Din • \\ Roberto Rossi • Alessia Tron • Alessandra Tellini • \\ Filippo Castoldi
}

Published online: 20 April 2012

(C) Springer Science+Business Media, LLC 2012

\section{Introduction}

Discovery consists of looking at the same thing as everyone else and thinking something different.

(Albert Szent-Györgyi de Nagyrápolt, 1893-1986; Nobel Prize in Medicine in 1937)

Since the introduction of tendoscopy for the treatment of peroneal tendinopathy in the late 1990s, orthopaedic surgeons have really "discovered" how to look closely at peroneal tendons and thus to "think of them in a different way," identifying new pathological entities and utilizing a modern, less-invasive technique to treat common pathologies. Indeed, diagnosis of lateral ankle pain is challenging, and clinical tests and imaging techniques like MRI often result in being inconclusive. Open surgery, on the other hand, allows for extensile exposure but implies postoperative scar formation and possible adhesions between tendons and their sheaths. Furthermore, functional evaluation is strongly impaired by incision of the peroneal sheath. Nervous structures, such as the sural nerve and superficial peroneal nerve, are also at risk in open procedures even when accurately identified because retraction of skin flaps and formation of scar tissue at the surgical site can cause neuritis and nerve entrapment. Theoretically, tendoscopy facilitates a minimally invasive approach that reduces the likelihood of postoperative scarring and nerve entrapment. Moreover, the whole tendon surface can be explored from the myotendinous junction to the peroneal tubercle, preserving the anatomical soft structures involved in tendon physiology and the intertendinous septum. In order to verify correct tendon gliding inside the fibro-osseous tunnel system, dynamic evaluation of peroneal tendons can also be performed under direct tendoscopic visualization by passive
Electronic supplementary material The online version of this article (doi:10.1007/s12178-012-9123-1) contains supplementary material, which is available to authorized users.

A. Marmotti $(\bowtie) \cdot$ M. Cravino $\cdot$ M. Germano $\cdot$ R. Del Din •

R. Rossi $\cdot$ A. Tron $\cdot$ A. Tellini $\cdot$ F. Castoldi

Department of Orthopaedics and Traumatology,

University of Turin,

Turin, Italy

e-mail: antonio.marmotti@inwind.it 
flexion and extension of the ankle. As a result, several pathological entities causing pain and tendon catching can be identified and treated, from simple tenosynovitis to peroneus quartus or hypertrophic peroneal tubercle. As arthroscopic procedures have became one of the major tools in diagnosis and treatment of intra-articular ankle lesions during the last decades, tendoscopy has been gradually incorporated into the "surgeon's armamentarium" [1•].

Literally, tendoscopy is "endoscopy of the tendon sheath." It was first proposed by Wertheimer in 1995 [2] for the treatment of posterior tibial tenosynovitis, and it was subsequently popularized for the treatment of different pathological entities involving posterior tibial tendon [3-5], Achilles tendon [6], flexor hallucis longus [7, 8], extensor digitorum longus [9], extensor hallucis longus [10], and tibialis anterior tendons [11]. Van Dijk first described tendoscopy of the peroneal tendon in 1998 [12] by performing a cadaver study followed by a prospective study on nine consecutive patients.

Visualizing tendons inside their sheath implies having familiarity with the course of the tendon. A thorough knowledge of the anatomy of peroneal tendons is therefore a prerequisite to performing tendoscopy. The peroneus longus muscle originates over the proximal two-thirds of the fibula, whereas the peroneus brevis muscle originates in a more distal orientation from the fibular shaft and interosseus membrane. Both muscles develop into tendons before reaching the ankle joint: the peroneus longus becomes completely tendinous at approximately $4 \mathrm{~cm}$ proximal from the tip of the fibula; the peroneus brevis usually has some muscle fibers that extend distally. However, as they run from the leg to the foot, they wrap around the lateral malleolus. Around this bony pulley, they lie in a fibro-osseous tunnel formed by the bone and the superior peroneal retinaculum that keeps the tendons in position [13]. Following the study of Sammarco [14•], four anatomical areas can be described: Zone A runs from the superior peroneal retinaculum to the apex of the distal fibula, and the peroneus brevis sits anteriorly to the peroneus longus. Zone B encompasses the area from the inferior peroneal retinaculum to the level of the peroneal tubercle (lateral wall of the calcaneus), and the peroneus longus runs inferior to the peroneus brevis. Tendons then separately enter the retinacular tunnels after the peroneal tubercle. Zone $\mathrm{C}$ is defined by the osseous groove in the plantar and lateral cuboid, where the peroneus longus curves beneath the cuboid. Zone D is represented by the tendon insertion at the metatarsal base where avulsion injury can be observed.

Two nervous structures are close to these areas. The superficial peroneal nerve comes out of the superficial fascia 7 to $14 \mathrm{~cm}$ proximal to the tip of the lateral malleolus, and it divides into the medial and intermediate dorsal cutaneous nerves at approximately $6 \mathrm{~cm}$ above the tip of the lateral malleolus $[15,16]$. This nerve and its branches are at risk of injury when introducing the tendoscope towards the muscletendon junction of the peroneus longus above zone A. The sural nerve is at risk when the tendoscopic distal portals are established in zone B, and it is rarely observed when crossing the surgical site upon performing dissection up to the tendon sheath.

In our experience, zones $\mathrm{A}$ and $\mathrm{B}$ are the only areas amenable to endoscopic exploration, so the indications for tendoscopy are reserved for pathological entities of these two proximal areas.

\section{Indications and surgical findings}

The main indication for peroneal tendoscopy is posterolateral ankle pain [13]. This condition may be associated with other symptoms deriving from intrarticular or extrarticular pathology such as lateral ankle instability, anterior impingement, chondral or osteochondral lesions, or subtalar malalignment (such as calcaneus varus). In this case, tendoscopy can be performed alone or as an accessory procedure together with other surgical techniques such as tibiotalar arthroscopy, anatomical reconstruction of the lateral compartment, or calcaneal osteotomy. Indeed, posterolateral ankle pain is often caused by disorders of the peroneal tendons. Table 1 summarizes the possible pathological entities that cause complaints at the postero-lateral side of the ankle.

Common clinical signs that are suggestive of peroneal tendon pathology are: lateral ankle pain with or without subjective instability, clicking, popping or snapping sensation during active eversion-inversion of the rearfoot, tenderness at palpation over the tendon course, edema or swelling at the posterolateral ankle over the tendon sheath, and an increase of pain on active eversion against manual resistance (positive resistance test) [12, 17].

MRI imaging usually confirms the diagnosis in severe cases of tendon tears and tenosynovitis, revealing fluid in the tendon sheath. However, it can also be inconclusive in more subtle cases that are associated with significant symptoms, for example, the presence of small peroneus quartus tendon $[17,18]$. For this reason, peroneal tendoscopy is indicated both when MRI scans reveal an abnormal signal in the peroneal tendons and when there is a strong clinical suspicion of peroneal tendinopathy with silent MRI [18].

The most frequent indication for peroneal tendoscopy is tenosynovitis [14•]. This is secondary to overuse or microtraumatic etiology, mainly in athletic patients, or to chronic mechanical irritation after ankle sprains, calcanear fractures, foot deformities, and rheumatic diseases [12]. Tendoscopy is indicated after at least 2 months of conservative management including ice, rest, shoe modifications, orthosis, 
Table 1 Main indications for peroneal tendoscopy and their treatment

\begin{tabular}{|c|c|c|}
\hline $\begin{array}{l}\text { Injury } \\
\text { site }\end{array}$ & Pathology & Proposed treatment \\
\hline \multirow{9}{*}{$\begin{array}{l}\text { Zone } \\
\text { A }\end{array}$} & Peroneal tenosynovitis & Tenosynovectomy (shaver) \\
\hline & Peroneal tendinopathy & $\begin{array}{l}\text { Tendon debridement and excision of the area of tendinosis } \\
\text { (shaver, mini-open repair) }\end{array}$ \\
\hline & $\begin{array}{l}\text { Hypertrophy of muscle belly (low-lying peroneus brevis } \\
\text { muscle belly) }\end{array}$ & Resection of the distal muscle fibers (shaver) \\
\hline & Partial tendon tears, longitudinal ruptures & Debridement (shaver, mini-open repair \\
\hline & Postsurgery or postfracture adhesions & Debridement (shaver) \\
\hline & $\begin{array}{l}\text { Bony prominences at the posteriori aspect of the fibula } \\
\text { (tendon sliding channel) }\end{array}$ & Excision (burr) \\
\hline & Tendon subluxation (instability) in shallow fibular groove & Groove deepening (burr) \\
\hline & $\begin{array}{l}\text { Peroneal impingement and subluxation in the presence of } \\
\text { accessory peroneal muscle (peroneus quartus) }\end{array}$ & Peroneus quartus excision (shaver; mini-open) \\
\hline & $\begin{array}{l}\text { Peroneal adhesion-snapping between tendons and their sheath } \\
\text { for thickened vinculum }\end{array}$ & Vinculum release (shaver) \\
\hline \multirow[t]{3}{*}{$\begin{array}{l}\text { Zone } \\
\text { B }\end{array}$} & $\begin{array}{l}\text { Peroneal impingement for hypertrophic or prominent peroneal } \\
\text { tubercle }\end{array}$ & Excision-flattening (burr) \\
\hline & Stenosis or degenerative tearing & $\begin{array}{l}\text { Resection of the impinging synovium, release of the inferior } \\
\text { retinaculum from the peroneal tubercle (shaver; mini-open) }\end{array}$ \\
\hline & Degenerative tendinopathy/tendon hypertrophy & Mini-open repair \\
\hline
\end{tabular}

kinesitherapy, and injection of corticosteroid and anaesthetic [13], the latter having both diagnostic and therapeutic values.

Panchbhavi and Trevino $[17,18]$ reported the presence of peroneus quartus muscle as a rare cause of postero-lateral ankle pain. This anomaly has an incidence reported between 13 and $21 \%$ and is frequently bilateral and more common in male patients. It can lead to swelling, tenderness, and instability of peroneal tendons, leading to a predisposition to intra-tendinous subluxation. It can be identified by MRI scan if the muscle is separated from the adjacent physiological peroneal structures. Similar symptoms arise due to a low-lying muscle belly of peroneus brevis, which remains muscular down to the level of the tip of the fibula. This condition is difficult to be identified by MRI but often highly symptomatic. Anatomic variations of the distal fibula may also lead to peroneal tendon subluxation and instability. These are the conditions characterized by a hypoplastic or absent osseous groove of the fibula, which occurs in $18 \%$ of the patients [19].

Some peroneal disorders, such as hypertrophic tendinopathy, vincula thickening, tenosynovitis, and tendon tears are often associated with chronic lateral ankle instability [12, 20]. This occurrence is due to the great strain on peroneal tendons during ankle inversion and eversion when proprioceptive afferents and passive stabilizers are insufficient, such as in anterolateral instability. Approximately $95 \%$ of patients undergoing ankle arthroscopy before ligamentous reconstruction had associated intra-articular pathology or cartilaginous damage, as observed by Komenda [21] and
Taga [22]; $25 \%$ of patients undergoing lateral ankle ligament reconstruction had previously been diagnosed with degenerative peroneus brevis tendon tears, as documented in a study by Di Giovanni [23]. Following these studies, routine tendoscopic or open evaluation of peroneal tendons during lateral ligament reconstruction should be proposed for every patient with suspicion of peroneal tendon pathology or postero-lateral ankle pain.

Finally, it should be mentioned that, by using tendoscopic techniques in 1998, van Dijk [12] first identified the anatomical structure called "peroneal vinculum" and, consequently, the thickening of this structure as a pathological entity. The peroneal vinculum is a membranous mesotendinous tissue connecting tendons at their sheaths. It is found between the peroneal longus and brevis tendons and is attached to the dorso-lateral aspect of the fibula. Peroneal vincula possess a vascular supply through their mesotendinous structure, previously related to having a trophic role and a healing response [24]. Mesotendinous thickening can become a source of morbidity, as it limits or tethers tendon excursion and may also impair clear visualization of the tendon. With the use of tendoscopy, a selective excision of the thickened portion of the vinculum can be performed in cases where symptoms such as pain and snapping are evident [25].

Differential diagnosis of postero-lateral ankle pain [13]:

- $\quad$ stress fractures around the fibula

- lesions of the lateral ligament complex

- posterior impingement of the ankle (os trigonum, flexor hallucis longus entrapment) 
- entrapment neuropathies of the sural nerve

- subtalar joint arthropathy

\section{Surgical technique}

Although tendoscopy uses the same concepts of all arthroscopic procedures, the difference between a joint space and the "virtual" space of the tendon sheath makes tendoscopic procedures quite complicated. Thus, certain surgical tips are fundamental to obtain a clear visualization of the tendon surface (Table 2). The first prerequisite is the availability of small joint instruments and a $30^{\circ}$ degree $2.7 \mathrm{~mm}$ arthroscope [1•]. The $4 \mathrm{~mm}$ arthroscope allows better visualization but is often difficult to introduce inside the sheath. The $2.7 \mathrm{~mm}$ scope is therefore the preferred tool to start tendon sheath examination. In rare instances, when treating pathologies at the fibular tip, a $2.7 \mathrm{~mm} 70^{\circ}$ scope can be useful in improving the visualization of the structure around the apex of the malleolar groove. For the same reason, only a $2.9 \mathrm{~mm}$ shaver can be introduced inside the peroneal sheath without jeopardizing its integrity. A 3.2 burr can be used to perform bone procedures, such as groove deepening or tubercle excision. A small probe is also useful to palpate the tendons and to measure the extent of any pathological entity such as tendon rupture or tendinosis.

Positioning of patients is disputed among different authors, as Sammarco [14•] advises a lateral decubitus position while van Dijk [13] proposes a supine placement. A reasonable compromise seems to be the semilateral decubitus, recommended when anterior ankle arthroscopy is associated. In this instance, the patient's body is tilted laterally (to nearly $45^{\circ}$ ), enabling easier access to the lateral side of the ankle without impairing the placement of the anteromedial tibiotalar portal. Tendoscopy should be performed before any other arthroscopic or open procedures to prevent any interference such as fluid extravasation at the lateral side of the ankle.

One of the most common difficulties is correctly establishing the first portal (Fig. 1a). This has to be done at 1.5$2 \mathrm{~cm}$ distally to the posterior edge of the apex of the fibula, just above the tendon sheath [12]. A small skin incision is made with a $\mathrm{n}^{\circ} 15$ scalpel and, with a "nick and spread" technique, the space between the subcutaneous tissue and the tendon sheath is spread bluntly with a small haemostat, which allows identification of any nervous structure crossing the surgical site and visualization of the external surface of the sheath. Then, with a curved Kelly clamp, the whole sheath is raised. Before introducing the scope, $20 \mathrm{cc}$ of saline solution are injected inside the sheath before incising it. This step causes the sheath to enlarge in the retromalleolar zone, facilitating the passage of the arthroscope. Then, a sharp longitudinal incision is performed with a $n^{\circ} 15$ scalpel along its lateral surface until the tendons are visualized, which allows for an easier introduction of the different tools without the risk of tearing the sheath. The scope is inserted inside the sheath under direct visualization and gently pushed upward. This step is easier if the ankle is plantarflexed, straightening the course of the peroneal tendons. This retrograde introduction of the scope also has a socalled "candle effect" ("effet bougie") [25] on the anatomical structures contained inside the sheath. The simple passage of the scope widens this space, leading to the lysis of any small adhesions. Nevertheless, major scar and stenosis need to be treated with shavers or small joint biters and knives.

The second portal can be established $2-2.5 \mathrm{~cm}$ proximal to the posterior edge of the lateral malleolus (Fig. 1b) and is guided by the light from the scope (namely "by transillumination") with the help of an 18 gauge spinal needle. During this step, it is advisable to keep the foot plantar flexed to straighten the axis of the peroneal tendons and to facilitate the introduction of the rigid scope into the superior peroneal retinaculum, allowing for a better visualization. Accessory portals can also be performed with this technique throughout the whole tendon sheath, according to the pathology that has to be treated, up to $6 \mathrm{~cm}$ proximal to the tip of the fibula [14•].

Once at least two portals are placed, the surgeon has to adopt a triangulation technique similar to that of subacromial space arthroscopy, positioning the instruments at an angle of nearly $180^{\circ}$ (Fig. 1). When the cause of the pain is identified, the surgeon can start the therapeutic procedure. Shifting the scope through the different portals, the shaver and the radiofrequency wand are used to remove scar tissue, hypertrophic synovial membrane, accessory muscles, and low-lying muscle belly, while the burr is used to excise or flatten any pathologic bony prominences [26•]. When a longitudinal rupture is observed (Fig. 1e), a small incision can be performed over the lesion site to allow for open tendon repair with a mini-open technique. (See supplementary files: Videos from Video 2 to Video 8 to view some of the previously described surgical steps).

\section{Postoperative program}

Postoperative management is strictly dictated by the cause of the pain and the intraoperative diagnosis. Routinely, it is advisable to keep the ankle in a removable brace to allow for early passive and active range of motion, preventing scar formation and adhesions. Weight-bearing is allowed according to the pain, usually after 3 weeks [18]. When mini-open tendon sutures or major tendon debridement are performed, the authors of this review suggest postponement of weight bearing until the fourth week postoperatively.

\section{Main complications}

The most common pitfall of tendoscopy is the rupture of the tendon sheath during the passage of surgical instruments, which causes a visual impairment due to the collapse of the 
Table 2 Surgical instrumentation and operative technique
Instrumentation

Patient positioning

Anesthesia

Tourniquet

Bony landmarks

Ankle position

Arthroscopy fluid

I portal (distal)

Inspection

Dynamic testing of the tendons

II portal (proximal)

III portal (accessory):

IV portal (accessory):

Fibular groove deepening

Suture $30^{\circ} 2.7$ short ("small joint") arthroscope

$30^{\circ} 4 \mathrm{~mm}$ arthroscope;

$70^{\circ} 2.7$ short ("small joint") arthroscope not always necessary

$2.9 \mathrm{~mm}$ shaver, $2 \mathrm{~mm}$ shaver, $3.2 \mathrm{~mm}$ burr

Small probe

Small joint knives and biters

$90^{\circ}$ small radiofrequency wand

supine, lateral decubitus, semilateral decubitus

general or epidural; in rare instances (diagnostic procedures) local anesthesia

around the proximal thigh, inflated to $120 \mathrm{~mm} \mathrm{Hg}$ above mean arterial pressure, after exsanguination and instillation of intravenous antibiotics

tip of the lateral malleolus, fifth metatarsal tuberosity, peroneal tubercle

plantar flexion and eversion

dilute epinephrine (1:1000) in normal saline to maintain hemostasis. Pressure of fluid: gravity feed or low flow pump system set at $50 \mathrm{~mm} \mathrm{Hg}$ or less to prevent insufflation of the subcutaneous tissue

$1.5-2 \mathrm{~cm}$ distal to the posterior edge of the apex of the fibula, just over the tendon sheath, proximal to the peroneal tubercle; blunt trocar with corresponding cannula introduced with saline irrigation; scope introduced inside the cannula

start $6 \mathrm{~cm}$ proximal from the posterior tip of the lateral malleolus where the fibrous septum splits the tendon compartment into two separate tendon chambers; more distally both tendons lie in one compartment; rotate the endoscope over and in between both tendons to complete the inspection; identify vincula if possible;

distal examination can be difficult due to the different tendon course and the small space

tendons through ankle flexion-extension and inversion-eversion; to test stability and exclude the presence of subluxation or dislocation

assisted by transillumination and with the help of an 18 gauge spinal needle; posterior to the fibula, $2-2.5 \mathrm{~cm}$ proximal to the tip of the fibula

distal to the myotendinous junction of the peroneus longus, $5-6 \mathrm{~cm}$ proximal to the tip of the fibula, $5 \mathrm{~mm}$ posterior to the palpable edge of the fibula

distal to the peroneal tubercle (for the release of the inferior peroneal retinaculum)

3.9 small burr introduced through the distal portal, endoscope through the proximal portal; typically the deepening has to be extended $3 \mathrm{~mm}$ in depth and $5 \mathrm{~mm}$ in width

Suture portals with non-absorbable sutures to prevent synovial shunt formation sheath and extravasations of the fluid [18]. Damage to peroneal tendons while entering the sheath is also possible, especially when large adhesions are present. Such an occurrence causes instruments to be "forced up" along the tendon surface. A simple piece of advice is to raise the fluid pressure inside the sheath, thus allowing for more space around the tendon body. Iatrogenic nerve injuries to both the superficial peroneal nerve (or its branches) above zone A or to the sural nerve, which can be very close to the distal portal, are also possible.

\section{Anatomic and histological study}

The authors performed the anatomic and histological study at the Institut d'Anatomie de l'Université Réné Descartes, Paris (France) and at the Department of Pathology, University of Torino, (Italy).

Aims of the study were:

1) To verify feasibility of peroneal tendoscopy in evaluating peroneal tendons (cadaveric study). 
Fig. 1 a "nick and spread" technique to establish the I distal portal; b II portal placement optimized by introducing a spinal needle; $\mathbf{c}$ view of peroneal tendons from distal to proximal; $\mathbf{d}$ tenosynovitis of the peroneal tendons; e traumatic longitudinal tear of peroneus brevis
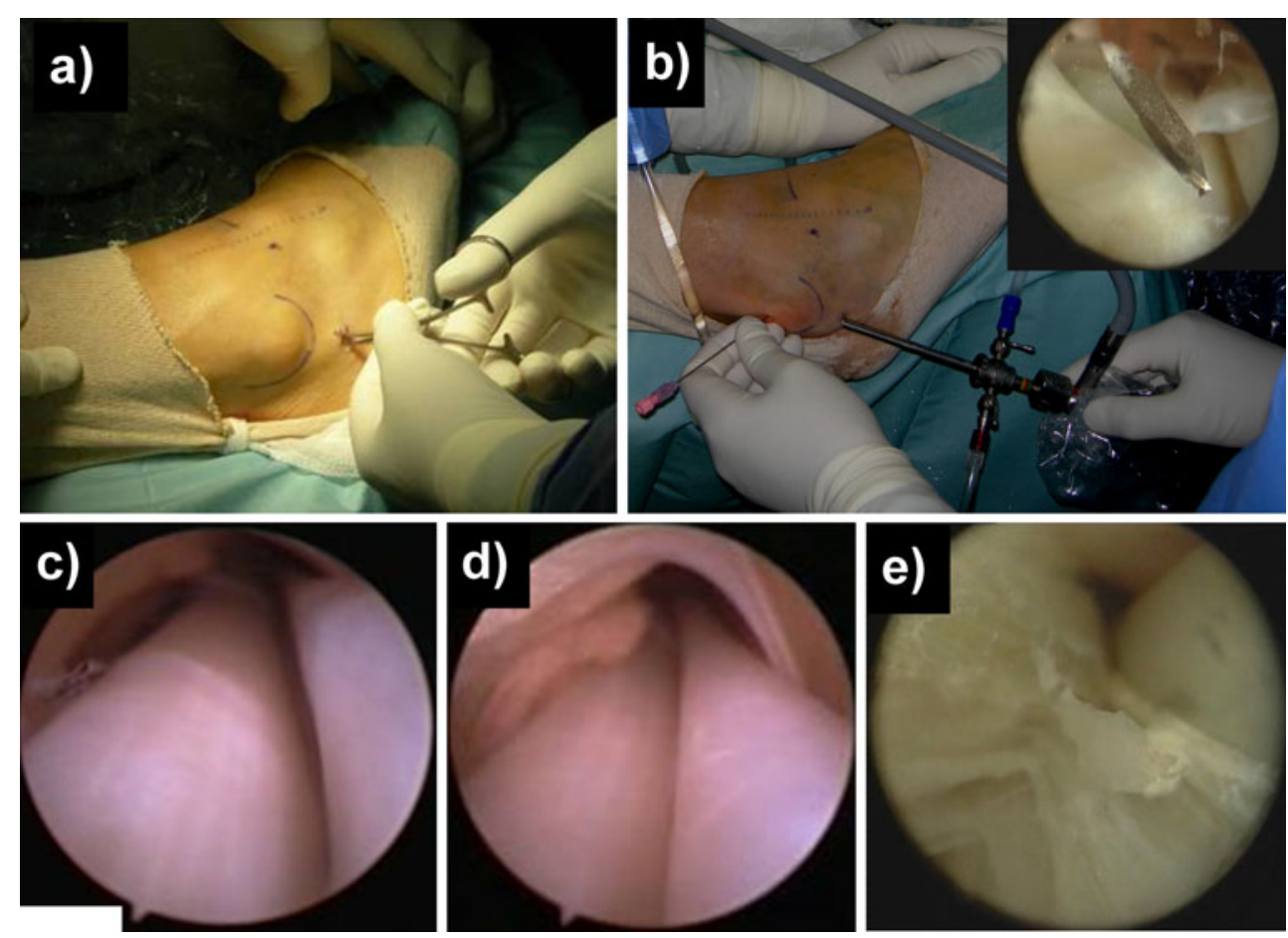

2) To verify the presence of nervous tissue in the peroneal vincula, allowing formulation of a hypothesis regarding their functional role (histological study).

\section{Materials and methods}

Cadaveric study (see supplementary files: Video 1: Cadaveric study)

Eight fresh-frozen ankles were used for the cadaveric study, with no previous history of ankle pathology. For each ankle, a distal portal was established following the previously described surgical technique. A $2.7 \mathrm{~mm}$ tendoscope was then introduced inside the peroneal sheath up to $7 \mathrm{~cm}$ above the apex of the fibula and left in place. Superficial dissection was performed and a skin flap was retracted anteriorly, exposing the tendon sheath and the superficial peroneal nerve with its branches. The distance between the sheath and the sural nerve was measured at the level of the distal portal. The distance between the apex of the endoscope and the nerve (or its closest branch) was measured at $6.4 \mathrm{~cm}$ proximal to the apex of the fibula. This is the point where the superficial peroneal often splits into two branches (namely: the medial dorsal cutaneous nerve and the intermediate dorsal cutaneous nerve) $[15,16]$. All measurements were performed using an electronic digital caliper by two independent observers; mean values were calculated. Deep dissection was then carried out and the peroneal sheath was sectioned longitudinally in order to expose the tendons.
Inspection of tendons was performed to verify the presence of both peroneal vincula.

\section{Histological study}

Samples of peroneal vincula were harvested from the retromalleolar groove (zone A), fixed in $10 \%$ neutral-buffered formalin, embedded in paraffin, and then cut with a microtome. Sections were evaluated by histology with Haematoxylin/Eosin staining (HE Sigma Chemical CoSt. Louis, MO) and by immunohistochemistry for S-100 (Abcam ab15520), p 75, also known as low-affinity neurotrophin receptor or NGFR (nerve growth factor receptor) or CD271(Abcam ab8874), myelin basic protein (MBP) (Abcam ab7349), PGP 9.5 (pan-neuronal marker protein gene product) (Novus Biological NB110-58874), Neurofilament (NF) (Abcam ab93340), and Vimentin, as undifferentiated markers for both nervous and vascular structures (Abcam ab8069). These choices were supported by several previous studies [27-37].

As positive controls, samples from the sural nerve and the sinus tarsi expansion of inferior extensor retinaculum were taken from each specimen, fixed in formalin, and evaluated as previously described for vincula samples. These samples were also compared to five peroneal vincula biopsies obtained from five patients affected by chronic ankle instability and undergoing tendoscopy for chronic lateral ankle pain. Tendoscopy was performed for persistent pain at the posterior margin of lateral malleolus after at least 4 months of non-operative treatment. Intraoperative findings 
of vincula lesion (thickening/scarring) were revealed during tendoscopy in all five patients, and a selective excision of the degenerated areas was performed. Biopsies were taken from the center of pathologic vincula and evaluated by histology with Haematoxylin/Eosin staining and by immunohistochemistry for S-100. An independent Pathologist examined all immunohistochemical specimens and a qualitative score of ++ or + was given to a strong positive staining or a weak positive staining, respectively.

\section{Results \\ Cadaveric study}

Placement of the distal portal was evaluated in relation to the distance from the sural nerve. The average distance was $3.9 \mathrm{~mm}$ (range 3.3-4.9 $\mathrm{mm}$ ) on dissection. There was no visible damage to the nerve while the portal was established. None of the anatomic dissections showed damage to the peroneal tendons during penetration of the instruments (cannula and scope) along the tendon sheath. The scope was easily inserted up to $7 \mathrm{~cm}$ proximal to the apex of the fibula. During this procedure, little resistance was observed while plantarflexing the ankle. After superficial dissection, the average distance of the superficial peroneal nerve to the apex of the endoscope was $14.7 \mathrm{~mm}$ (range 13-16.5 mm). In all specimens, vincula were observed as thin membranous mesotendineal structures. Peroneus longus vinculum was more prominent throughout the course of the tendon, while that of the peroneus bevis was thinner and absent in the retro-inframalleolar area behind the fibular groove in five out of eight cases.

Histological study (See Fig. 2 and Supplement 1: Peroneal tendoscopy histological study)

Immunohistochemistry of cadaveric vincula was positive for the presence of nervous fibers in all specimens. Stronger signals were observed with anti S-100 and anti-NF antibodies, while the weakest signal was given by antibodies against NGFR (p75). Neither Ruffini endings nor Pacinian corpuscles were clearly observed due to the fact that Pacinilike corpuscles are usually identified through their thick perineurial capsules with marked p75 immunostaining, while these specimens only showed weak positivity for p75. Instead, the immunohistochemical pattern was consistent with the presence of type IV free nerve fibers, either present as being isolated inside the membranous structure of peroneal vincula or in the proximity of tendon bodies or vessels. All patients' biopsies from scarred vincula were positive when labeled with anti S-100 antibodies. Free

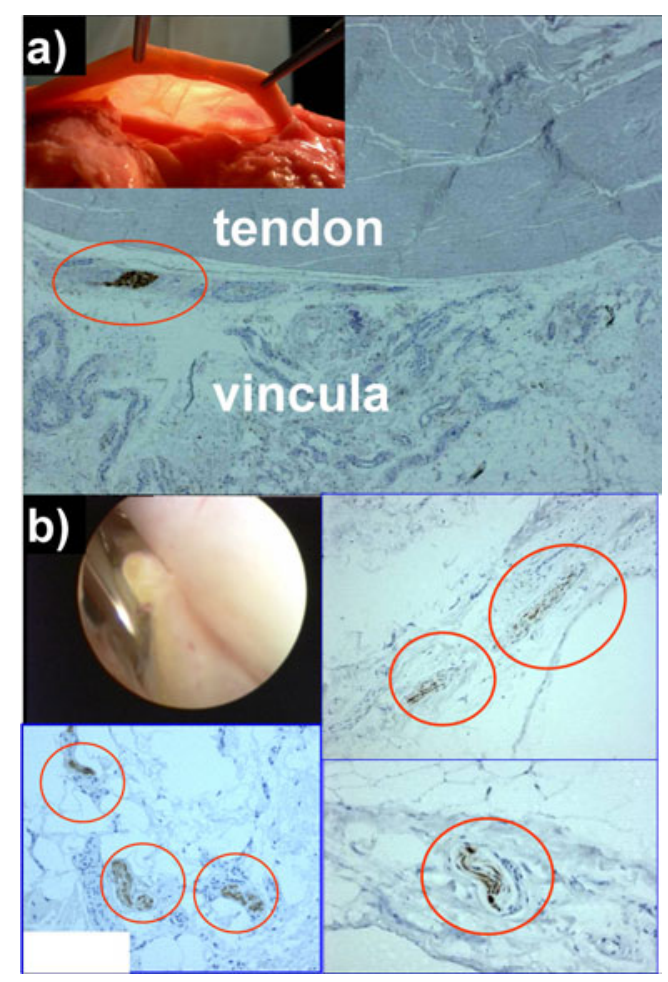

Fig. 2 Immunohistochemistry for S-100 protein in vincula specimens; a cadaveric vincula sample; b vinculum biopsy harvested during tendoscopic release of a thickened vincula sample

nervous fibers were recognizable with a pattern similar to type IV free nerve endings. All patients reported subjective improvement of lateral pain upon clinical examination 2 months after tendoscopic excision of the thickened portion of the vincula. Positive results at immunohistochemistry were also obtained with specimens of the sural nerve and extensor retinaculum (positive controls) (Table 3).

\section{Conclusion}

Extra-articular tendon pathologies of the ankle were traditionally treated with open surgery. The number of arising complications, such as nerve injuries, infections, postoperative pain, scarring, and stiffness around the ankle joint, encouraged the surgeons to apply endoscopic techniques in foot and ankle surgery. Tendoscopy is an appealing and fascinating solution to the above limitations. Peroneal tendons are good candidates for tendoscopic treatment because of their subcutaneous position along the lateral wall of the calcaneus and the posterolateral side of the fibula. Indeed, tendoscopy allows for a unique view of the entire length of peroneal tendons while also providing a dynamic evaluation of their movement inside the sheath, and it is a useful tool both for the diagnosis and the minimally invasive treatment of different peroneal tendon disorders. 
Table 3 Immunohistochemical analysis of specimens (cadaveric vincula, vincula from patients' biopsies, sural nerve, extensor retinaculum)

\begin{tabular}{llllll}
\hline specimen & S100 & p 75 & MBP & PGP 9.5 & vimentin \\
\hline Cadaveric peroneal vincula & ++ & + & ++ & ++ & ++ \\
Vincula from patients' biopsies & ++ & $/$ & $/$ & $/$ & $/$ \\
Sural nerve & ++ & ++ & ++ & $/$ & $/$ \\
Inferior extensor retinaculum & ++ & ++ & ++ & + & ++ \\
\hline
\end{tabular}

S-110 = S-100 protein is a family of low molecular weight proteins found in vertebrates, characterized by two calcium binding sites of the helixloop-helix ("EF-hand type") conformation; the name is derived from the fact that the protein is $100 \%$ soluble in ammonium sulfate at neutral $\mathrm{pH}$ p75. = low-affinity neurotrophin receptor, NGFR, nerve growth factor receptor, CD271

$\mathrm{MBP}=$ myelin basic protein

PGP $9.5=$ pan-neuronal marker protein gene product

$\mathrm{NF}=$ neurofilament

Vimentin = type III intermediate filament (IF) protein that is expressed in all mesenchymal cells; here it is used as an undifferentiated marker for both nervous and vascular structures

$++=$ strong positive staining at immunohistochemistry

$+=$ weak positive staining at immunohistochemistry

/ = staining not performed

Advantages of tendoscopy are common to other arthroscopic and less invasive endoscopic procedures in ankle surgery. It can be performed as an out-patient procedure or as a "one-day surgery" hospitalization. Surgical morbidity and postoperative pain are reduced when compared to open procedures. Endoscopic treatment of tendon pathologies has the potential to reduce recovery time, wound infections, skin sloughing, and painful scars. Thus, earlier range of motion recovery, quicker work resumption, and a faster return to sporting activities can be hypothesized after a tendoscopic procedure. It also has the theoretical advantage to be performed under local anesthesia as a diagnostic procedure [12] in very select and compliant patients with unexplained symptoms. In these instances, tendoscopy may reveal the underlying pathology previously undetectable with common ultrasonography or MRI, as in lateral pain associated with snapping during active movement due to inter-tendinous subluxation, or lateral pain in forced eversion due to partial rupture of a peroneal tendon or scarring of the vincula.

The main disadvantage of this procedure is that it can be technically demanding in patients with extensive tenosynovitis or a scarred/thickened peroneal sheath. As a consequence, it might be necessary to convert the tendoscopy into an open procedure, which can happen whenever the lack of volume inside the sheath impairs a clear visualization and the safe passage of the surgical instruments, as in advanced scarification, stenosing tenosynovitis, presence of bulky accessory muscles, or bony prominences. In the future, this method could be partially improved by the use of flexible tools that might allow easier mobility and rotation of the scope to obtain a wider visual angle.

Similarly to previous articles $[12,13]$, the authors performed an anatomic study to evaluate both the theoretical feasibility of the tendoscopic technique and the histological structure of the new entity called "peroneal vincula." Based on the data of the first part of this study, the tendoscope appears to be easily passed through the entire length of the tendon sheath, theoretically with little risk of damaging the sural nerve or the superficial peroneal nerve due to the consistent distance measured with these nervous structures. Therefore, this technique seems to be relatively safe in the treatment of peroneal tendon diseases. A weakness of this part of the study is that measurements obtained during dissection may have been influenced by movement of the cannula and anatomic structures, though this might be counterbalanced by the fact that no gross nerve injuries from the cannula were identified during the anatomical dissection in all specimens. In the future, a clinical study might fully ascertain the safety of this procedure in order to prevent major damage to the surrounding neurovascular structures.

In the second part of the anatomical study, the authors evaluated the possible presence of nervous structures inside the vincula, both on cadaveric specimens and on biopsies from scarred vincula in patients affected by lateral ankle instability. Previous literature has provided no data about innervation of peroneal vincula. This is the first study to show the presence of free nervous fibers inside vincula, both in previously healthy specimens and in scarred vincula associated with the clinical picture of lateral ankle pain in chronic ankle instability. No clear differences between the two groups have been observed, although the comparison has only been based on the H/E staining and S100 immunohistochemistry. However, the better quality and greater quantity of the cadaveric tissue facilitated execution of multiple analyses with different nerve tissue markers, as shown in Table 3. The choice of these markers was based 
on the previously cited studies in which the immunohistochemical markers S-100, p75, PGP9.5, MBP, and NF allowed mechanoreceptors and nerve fibers to be identified. These analyses confirmed the presence of nervous structures similar to type IV nerve endings throughout the whole vinculum. These observations suggest a possible proprioceptive role of the vinculum similar to previous studies on the innervation of different anatomic structures [34, 36, 37]. Indeed, peroneal vincula might be part of the peritalar proprioceptive system, together with tibiotalar and subtalar joint capsules, inferior extensor retinaculum (with its expansion in the sinus tarsi), and medial and lateral ankle ligaments. Furthermore, post-traumatic lesions of the vincula, as observed in chronic lateral ankle instability, could be linked to painful symptoms experienced by patients [34]. Due to the presence of free sensitive and proprioceptive free nervous fibers, a thickened and scarred peroneal vinculum may become a nociceptive source. This occurrence makes the injured vincula an element concurring to proprioception impairment and chronic pain observed in clinical pictures of chronic ankle instability, together with other chronically injured tissues, such as the joint capsule and lateral ligaments. In the authors' personal opinion, this may have a clinical relevance to expand the indications for tendoscopic procedures. Indeed, in patients complaining of lateral ankle pain and chronic ankle instability, tendoscopy could be routinely performed prior to ligament reconstruction. This undertaking would allow evaluation of peroneal vincula and, in the evidence of thickened and scarred tissue, performance of a selective endoscopic excision of the degenerated areas of the vinculum, as an accessory procedure in the treatment of symptomatic chronic ankle instability.

Acknowledgments The Authors are grateful to Luciano Mosso (MD, Pathologist) for the histological assistance and the critical observations. The Authors would also like to thank Radhika Srinivasan, $\mathrm{PhD}$, for editing of the manuscript.

Disclosure No conflicts of interest relevant to this article were reported.

\section{References}

Papers of particular interest, published recently, have been highlighted as:

- Of importance

1. Christensen JC, Lanier TD. Tendoscopy of the ankle. Clin Podiatr Med Surg. 2011;28:561-70. This is the most recent review about Tendoscopy of the Ankle. It is a comprehensive overview of all the possible application of tendoscopy in ankle surgery including peroneal tendon diseases, posterior tibial tendon and Achilles tendon pathologies, flexor hallucis longus disorders.
2. Wertheimer SJ, Weber CA, Loder BG, et al. The role of endoscopy in treatment of stenosing posterior tibial tenosynovitis. J Foot Ankle Surg. 1995;34:15-22.

3. van Dijk CN, Kort N, Scholten PE. Tendoscopy of the posterior tibial tendon. Arthroscopy. 1997;13:692-8.

4. van Sterkenburg MN, Haverkamp D, van Dijk CN, Kerkhoffs GMMJ. A posterior tibial tendon skipping rope. Knee Surg Sports Traumatol Arthrosc. 2010;18:1664-6.

5. Chow HT, Chan KB, Lui TH. Tendoscopic debridement for stage I posterior tibial tendon dysfunction. Knee Surg Sports Traumatol Arthrosc. 2005;13:695-8.

6. Steenstra F, van Dijk CN. Achilles tendoscopy. Foot Ankle Clin. 2006;11:429-38. viii.

7. van Dijk CN. Hindfoot endoscopy. Foot Ankle Clin. 2006;11:391414. vii.

8. Lui TH. Flexor hallucis longus tendoscopy: a technical note. Knee Surg Sports Traumatol Arthrosc. 2009;17:107-10.

9. Lui TH. Extensor tendoscopy of the ankle. Foot Ankle Surg. 2011;17:e1-6.

10. Lui TH. Arthroscopically assisted Z-lengthening of extensor hallucis longus tendon. Arch Orthop Trauma Surg. 2007;127:855-7.

11. Maquirriain J, Sammartino M, Ghisi JP, Mazzuco J. Tibialis anterior tenosynovitis: avoiding extensor retinaculum damage during endoscopic debridement. Arthroscopy. 2003;19:E9.

12. van Dijk CN, Kort N. Tendoscopy of the peroneal tendons. Arthroscopy. 1998;14:471-8.

13. Scholten PE, van Dijk CN. Tendoscopy of the peroneal tendons. Foot Ankle Clin. 2006;11:415-20. vii.

14. - Sammarco VJ. Peroneal tendoscopy: indications and techniques. Sports Med Arthrosc. 2009;17:94-9. This paper is a complete and accurate review about the indications and techniques of peroneal tendoscopy written by one of the most know foot and ankle surgeons in United States.

15. Sarrafian SK. Anatomy of the Foot and Ankle. Philadelphia: Lippincott Williams and Wilkins; 1993.

16. Blair JM, Botte MJ. Surgical anatomy of the superficial peroneal nerve in the ankle and foot. Clin Orthop Relat Res. 1994;229-238.

17. Panchbhavi V. Peroneal tendoscopy and report on anomalies diagnosed. Foot Ankle Surg. 2003;9:131-5.

18. Panchbhavi VK, Trevino SG. The technique of peroneal tendoscopy and its role in management of peroneal tendon anomalies. Techniques in Foot \& Ankle Surgery. 2003;2:192-8.

19. Edwards ME. The relations of the peroneal tendons to the fibula, calcaneus, and cuboideum. Am J Anat. 1928;42:213-53.

20. Sobel M, Geppert MJ, Warren RF. Chronic ankle instability as a cause of peroneal tendon injury. Clin Orthop Relat Res. 1993;187-191.

21. Komenda GA, Ferkel RD. Arthroscopic findings associated with the unstable ankle. Foot Ankle Int. 1999;20:708-13.

22. Taga I, Shino K, Inoue M, et al. Articular cartilage lesions in ankles with lateral ligament injury. An arthroscopic study. Am J Sports Med. 1993;21:120-6. discussion 126-127.

23. DiGiovanni BF, Fraga CJ, Cohen BE, Shereff MJ. Associated injuries found in chronic lateral ankle instability. Foot Ankle Int. 2000;21:809-15.

24. Sobel M, Geppert MJ, Hannafin JA, et al. Microvascular anatomy of the peroneal tendons. Foot Ankle. 1992;13:469-72.

25. Dumontier C. Chirurgie endoscopique des membres. e-mémoires de l'Académie Nationale de Chirurgie. 2004;3:34-44.

26. - Jerosch J, Aldawoudy A. Tendoscopic management of peroneal tendon disorders. Knee Surg Sports Traumatol Arthrosc. 2007;15:806-10. This recent prospective study included 15 patients with chronic peroneal tendon dysfunction. It offers clear pictures about different pathologic conditions along with their treatment.

27. Alpantaki K, McLaughlin D, Karagogeos D, et al. Sympathetic and sensory neural elements in the tendon of the long head of the biceps. J Bone Joint Surg Am. 2005;87:1580-3. 
28. Bagge J, Lorentzon R, Alfredson H, Forsgren S. Unexpected presence of the neurotrophins NGF and BDNF and the neurotrophin receptor $\mathrm{p} 75$ in the tendon cells of the human Achilles tendon. Histol Histopathol. 2009;24:839-48.

29. Hagert E, Garcia-Elias M, Forsgren S, Ljung B-O. Immunohistochemical analysis of wrist ligament innervation in relation to their structural composition. J Hand Surg Am. 2007;32:30-6.

30. Lee J, Ladd A, Hagert E. Immunofluorescent Triple-Staining Technique to Identify Sensory Nerve Endings in Human Thumb Ligaments. Cells Tissues Organs 2011, In Press.

31. Liang Y, Johansson O. Light and electron microscopic demonstration of the p75 nerve growth factor receptor in normal human cutaneous nerve fibers: new vistas. J Invest Dermatol. 1998;111:114-8.

32. Keller K, Nasrilari M, Filler T, Jerosch J. The anterior tibio-talar ligament: one reason for anterior ankle impingement. Knee Surg Sports Traumatol Arthrosc. 2010;18:225-32.
33. Dalsgaard CJ, Björklund H, Jonsson CE, et al. Distribution of neurofilament-immunoreactive nerve fibers in human skin. Histochemistry. 1984;81:111-4.

34. Kiter E, Karaboyun T, Tufan AC, Acar K. Immunohistochemical demonstration of nerve endings in iliolumbar ligament. Spine. 2010;35:E101-104.

35. Gonzalez-Martinez T, Perez-Piñera P, Díaz-Esnal B, Vega JA. S100 proteins in the human peripheral nervous system. Microsc Res Tech. 2003;60:633-8.

36. Del Valle ME, Harwin SF, Maestro A, et al. Immunohistochemical analysis of mechanoreceptors in the human posterior cruciate ligament: a demonstration of its proprioceptive role and clinical relevance. J Arthroplasty. 1998;13:916-22.

37. Dhillon MS, Bali K, Vasistha RK. Immunohistological evaluation of proprioceptive potential of the residual stump of injured anterior cruciate ligaments (ACL). Int Orthop. 2010;34:737-41. 